\title{
Painless wrist lump
}

\author{
Rupert Berkeley ${ }^{1} \cdot$ Daniel Lindsay $^{2} \cdot$ Rob Pollock $^{3} \cdot$ Asif Saifuddin $^{1}$
}

Received: 27 October 2020 / Revised: 7 December 2020 / Accepted: 13 December 2020 / Published online: 21 January 2021

(C) ISS 2021

\section{Diagnosis}

Parosteal osteosarcoma of the distal radius

\section{Discussion}

Radiographs showed an extra-osseous, well-defined ossified mass arising from the volar, distal radial metadiaphysis with a lobular 'cauliflower-like' morphology. There was adjacent cortical thickening but no overt cortical destruction or aggressive periosteal reaction (Fig. 1). CT showed stippled mineralisation extending from the volar aspect of the cortex with medullary continuity (Fig. 2). The lesion had a broad base and was more ossified centrally than peripherally, with no appreciable soft tissue mass or cortical destruction. MRI demonstrated a dome-shaped mass measuring $14 \times 12 \times$ $10 \mathrm{~mm}$ arising from the volar radial cortex (Fig. 3). This was of heterogeneous low T1W SI and heterogenous intermediateto-low T2W SI centrally with thin peripheral hyperintensity suggestive of a poorly defined cartilage cap. Involvement of the cortex was optimally demonstrated on the post-contrast fat-suppressed T1W TSE image sequences with minor underlying marrow abnormality suggestive of early medullary involvement. The lesion was considered likely benign, with bizarre parosteal osteochondromatous proliferation (BPOP)

The case presentation can be found at https://doi.org/10.1007/s00256020-03698-0

Rupert Berkeley

rupert.berkeley@nhs.net

1 Department of Radiology, Royal National Orthopaedic Hospital, Brockley Hill, Stanmore, Middlesex HA7 4LP, UK

2 Department of Pathology, Royal National Orthopaedic Hospital, Brockley Hill, Stanmore, Middlesex HA7 4LP, UK

3 Department of Surgery, Royal National Orthopaedic Hospital, Brockley Hill, Stanmore, Middlesex HA7 4LP, UK the initial diagnosis. Excision biopsy was therefore performed. Histopathology sections showed a tumour composed of anastomosing trabeculae of lamellar and woven bone with intervening mildly atypical fibroblastic spindle cells arranged in vague fascicles and embedded in a densely collagenous stroma. Occasional mitoses were identified (3/10HPF) with no atypical forms present. The tumour also had an incomplete cartilaginous cap with no high-grade cytological atypia identified (Fig. 4). Fluorescence in situ hybridisation showed $M D M 2$ amplification and therefore the tumour showed classical features of a low-grade parosteal osteosarcoma.

Repeat MRI obtained 4 weeks post-operatively (Fig. 5) demonstrated residual surface and medullary tumour. Completion staging with whole-body MRI and CT chest showed no metastases, and the patient subsequently underwent a 'shark-bite' re-excision 6 weeks after the original operation; the resection specimen is demonstrated in Fig. 6.

Parosteal osteosarcoma is the commonest surface osteosarcoma, accounting for $4 \%$ of osteosarcomas [1]. Unlike conventional osteosarcoma, there is a slight predilection for females and an older age range, with a median age at presentation in the 4th decade $[1,2]$. It is a malignant but low-grade tumour with dedifferentiation being observed in approximately $25 \%$ of cases, most commonly to high-grade surface osteosarcoma or spindle cell sarcoma [3]. Medullary involvement on cross-sectional imaging is reported to correlate with the degree of dedifferentiation [2, 4], although reactive remodelling of underlying bone may cause confusing imaging appearances [5]. Survival rates are higher than for conventional osteosarcoma [1].

The femur is the commonest site affected (approximately two-thirds of cases and usually in the distal metaphysis), followed by the humerus and tibia, these 3 locations accounting for $>90 \%$ of cases $[2,4]$. The radius is an extremely unusual site, accounting for only $1.5 \%$ of cases $[2,4]$. The classical radiographic description is of a lobular, densely ossified mass with a broad, sclerotic cortical base. The 'string sign', a thin radiolucency interposed between the tumour and native cortex, is seen in only a minority of cases [6]. Conventional MRI 
Fig. 5 Post-operative MRI of the right distal forearm. (a) Sagittal T2W FSE and (b) axial PDW FSE MR images demonstrate residual intramedullary tumour (black arrows) which has grown since the initial MRI study 4 months previously, as well as soft tissue swelling at the surgical site (white arrowheads)
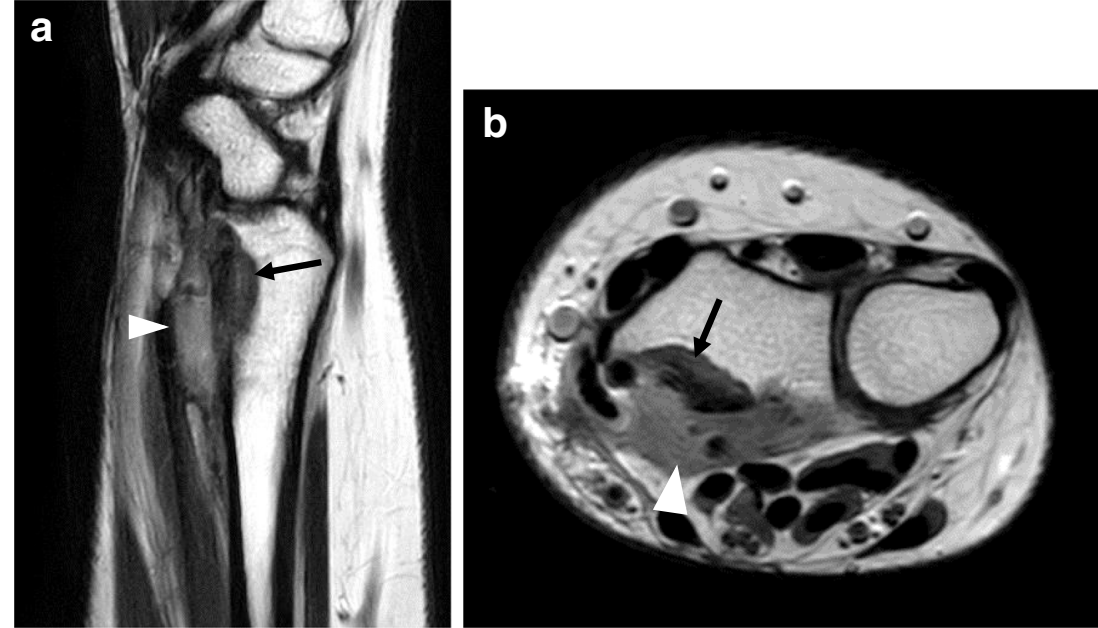

sequences show low signal intensity similar to cortical bone due to pronounced mineralisation, while regions of soft tissue signal intensity suggest dedifferentiated tumours.

Multiple differential diagnoses exist. Parosteal osteosarcoma may mimic the exophytic ossification of heavily calcified BPOP, an entity which also only rarely arises from the distal forearm long bones [7] and rarely involves the medullary cavity. A history of trauma may suggest florid reactive periostitis, but this is usually associated with prominent soft tissue oedema. The pattern of mineralisation in myositis ossificans is usually the inverse of parosteal osteosarcoma, with peripheral ossification classically described. Parosteal osteoma is a very rare entity, characterised by uniformly dense parosteal bone formation separated from the medullary cavity by cortex which may be normal or thickened [8]. Osteochondroma is usually distinguishable by demonstrating both medullary continuity with the central portion of the lesion and a cartilage cap [9]. Malignancies with

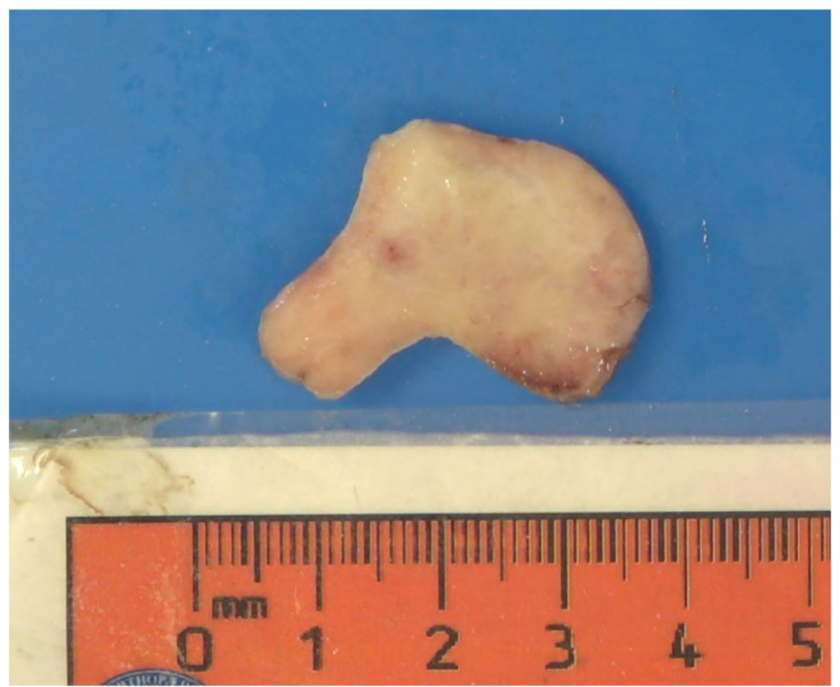

Fig. 6 Photograph of the cut surface of the second resection specimen showing a smooth, lobulated piece of bony tissue with a pale, homogenous cut surface. The macroscopic features are relatively nonspecific mineralised extra-osseous components such as conventional osteosarcoma and chondrosarcoma should also be considered, given their relative propensity for the long bones [10].

Management options for distal radial surface tumours vary according to the aggressiveness of the lesion. Low-grade surface tumours are typically treated with a 'shark-bite' excision which removes the surface tumour and the intramedullary extension, usually with no requirement for reconstruction of the bone. High-grade malignant tumours require wide resection and reconstruction with a vascularised fibular strut graft, possibly with neoadjuvant/adjuvant chemotherapy depending upon the diagnosis and the age of the patient.

The identification of a distal radial surface lesion should prompt careful clinical history and image interrogation since a variety of pathologies can be encountered. In the absence of classical features that convincingly point to one of the more common diagnoses there should be a low threshold for referral to a specialist centre.

\section{Compliance with ethical standards}

Conflict of interest The authors declare that they have no conflict of interest.

\section{References}

1. Damron TA, Ward WG, Stewart A. Osteosarcoma, chondrosarcoma, and Ewing's sarcoma: National Cancer Data Base Report. Clin Orthop Relat Res. 2007;459:40-7.

2. Ruengwanichayakun P, Gambarotti M, Frisoni T, Gibertoni D, Guaraldi F, Sbaraglia M, et al. Parosteal osteosarcoma: a monocentric retrospective analysis of 195 patients. Hum Pathol. 2019;91:11-8.

3. Bertoni F, Bacchini P, Staals EL, Davidovitz P. Dedifferentiated parosteal osteosarcoma: the experience of the Rizzoli Institute. Cancer. 2005;103(11):2373-82.

4. Okada K, Frassica FJ, Sim FH, Beabout JW, Bond JR, Unni KK. Parosteal osteosarcoma. A clinicopathological study. J Bone Joint Surg Am. 1994;76(3):366-78. 
5. Shimoyama T, Yamamoto Y, Kuroda M, Takakuwa Y, Washimi Y, Ishimura $\mathrm{D}$, et al. Cortical bone remodeling in parosteal osteosarcoma mimicking medullary involvement: a case with the difficulty in presurgical staging. Tohoku J Exp Med. 2014;233(3):165-9.

6. Smith J, Ahuja SC, Huvos AG, Bullough PG. Parosteal (juxtacortical) osteogenic sarcoma. A roentgenological study of 30 patients. J Can Assoc Radiol. 1978;29(3):167-74.

7. Washington E, Menendez L, Fedenko A, Tomasian A. Bizarre parosteal osteochondromatous proliferation: rare case affecting distal ulna and review of literature. Clin Imaging. 2020;69:233-7.

8. Bertoni F, Unni KK, Beabout JW, Sim FH. Parosteal osteoma of bones other than of the skull and face. Cancer. 1995;75(10):246673.
9. Sanghavi S, Wahegaonkar A, Panchwagh Y, Kharat A. Parosteal osteosarcoma of the distal radius mimicking an osteochondroma-a diagnostic misadventure. J Hand Surg Am. 2017; 42(12): 1038.e1031-1038.e1010.

10. Harper K, Sathiadoss P, Saifuddin A, Sheikh A. A review of imaging of surface sarcomas of bone. Skeletal Radiol. 2020.

Publisher's note Springer Nature remains neutral with regard to jurisdictional claims in published maps and institutional affiliations. 